\title{
Study on Characteristics of Spatial Distribution of Restaurants in Built-up Area of Chuxiong City
}

\author{
Wujun Xi ${ }^{1, a}$, Renjuan Luo ${ }^{2, b}$ \\ ${ }^{1}$ School of Geography and Tourism Management, Chuxiong Normal University, Chuxiong, China \\ ${ }^{2}$ School of Tourism and Geography Science, Yunnan Normal University, Kunming, China \\ aabsxwj@163.com, b664611530@qq.com
}

\begin{abstract}
Keywords: Spatial Distribution, Restaurants, K-means Clustering Method, Buffer Analysis, Chuxiong City.

Abstract. In order to obtain characteristics of spatial distribution of restaurants in built-up area of Chuxiong city, the paper used average center, median center, center element, standard distance, direction distribution, k-means clustering method and buffer analysis to analyze it, then these conclusions could be drawn as follows. (1) The positions of the average center, median center and center element were located in the west of lucheng north road, north of lucheng west road, east of longhui road, and south of Zixi avenue in the old city zone. The center element was Kunming jianxin garden crossing-over bridge rice noodles shop (chuxiong shop). (2) Space distribution of restaurants in built-up area of Chuxiong city was discrete, they did not significantly concentrate in one place or a small area. (3) Direction distribution of restaurants was northwest - southeast in built-up area of Chuxiong city. (4) It could be found that most of the restaurants centered around roads, while, crowding level of lanes and residential areas was relatively low with k-means clustering method and buffer analysis.
\end{abstract}

\section{Introduction}

Restaurants provide residents with a variety of food and beverage service. They are important facilities in each city. Especially with the accelerating pace of life, people's daily diet more and more depends on restaurants. In addition, with the continuous development of tourism in chuxiong city, diet as one of the important tourism activities, tourists demand for catering service level has been improved. Therefore, the reasonableness of spatial distribution of restaurants will influence the evaluation of the whole city from citizens and foreign tourists.

For the directional distribution analysis of restaurants, domestic related research mainly focused on the star grade hotel, research areas include the national scale, For example, the study on star grade hotel in the provinces[1,2], also had some studies on a region or cities, such as Chu Chiang delta[3], Shanghai city[4], Chongqing city[5], Nanjing city[6], Guilin city[7,8], Hefei city[9], Chunan county[10], etc. The research content mainly included spatial distribution and influencing factors, research methods are mainly traditional qualitative analysis $[9,10]$ and quantitative analysis, such as buffer analysis[7], standard deviation, coefficient of variation, correlation analysis[1,4], spatial syntax[8], etc.

"Restaurants" in this paper included restaurants and beverages shop. On the basis of previous studies, this paper mainly analyzed the characteristics of spatial distribution of restaurants in built-up area of Chuxiong city with average center, median center, center element, standard distance, direction distribution, clustering analysis, buffer analysis.

\section{The Number of Restaurants in Built-up Area of Chuxiong City}

There were 492 restaurants in built-up area of Chuxiong city, among them there were 219 restaurants in development zone, 273 restaurants in the old city zone. Development zone covers an area of 5.8691 $\mathrm{km} 2$, the old city zone covers an area of $18.2728 \mathrm{~km} 2$. Through calculation, it was known that the number of restaurants in development zone was 37.31 per square kilometer and the number of 
restaurants in the old city zone was 14.94 per square kilometer. The number of restaurants density of the development zone is 2.50 times of the number of restaurants density of the old city zone.

\section{Characteristics of Spatial Distribution of Restaurants in Built-up Area of Chuxiong City}

The spatial distribution of restaurants in built-up area of Chuxiong city was shown in figure 1, the linear features in the Figure 1 was longchuanjiang river, which divided built-up area of Chuxiong city into development zone (in the north, smaller area) and old city zone (in the south, larger area ). It could be seen that restaurants in development zone mainly concentrated in the northern area of longchuanjiang river, however, restaurants in the old city zone mainly scattered in the old city zone.

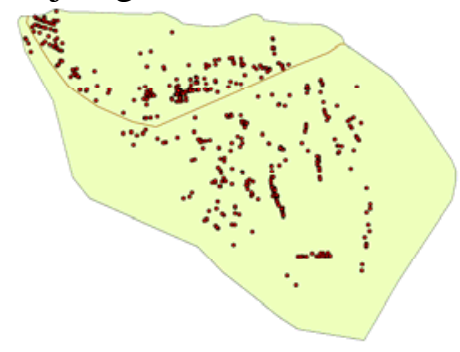

Figure 1 Spatial Distribution of Restaurants in Built-up Area of Chuxiong City

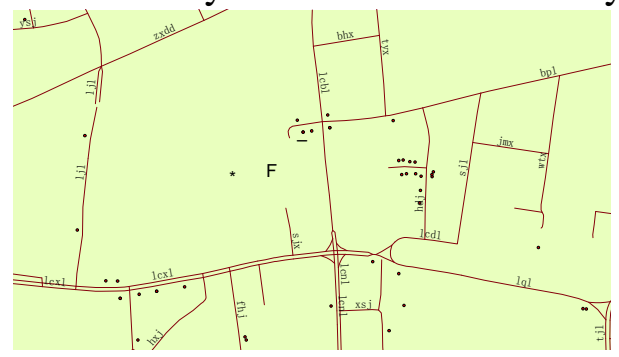

Figure 2 The Position of Average Center, Median Center, Center Element of Restaurants in Built-up Area of Chuxiong City

In order to accurately measure characteristics of spatial distribution of restaurants in built-up area of Chuxiong city, we used five indexes (average center, median center, center element, standard distance, direction distribution) to calculate it. The software we used was ArcMAP10.2.

In figure 2, $\triangle$ was the position of the average center, क was the position of the median center, is was the position of the center element. It could be seen that they were located in the west of lucheng north road, north of lucheng west road, east of longhui road, and south of Zixi avenue in the old city zone. The center element was Kunming jianxin garden crossing-over bridge rice noodles shop (Chuxiong shop). This zone was the nuclear region of the old city zone. It was close to the main commercial facilities(such as luchen building, people's shopping mall, wal-mart, gome, suning, etc), main parks(such as taoyuan Hu square, longjiang park), which had a large flow of people.

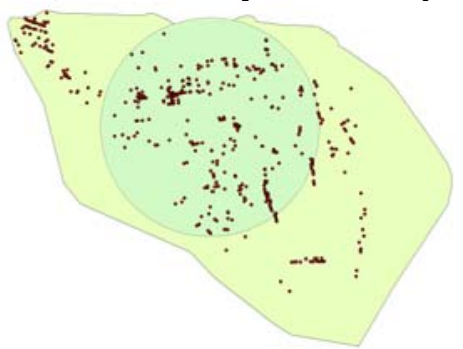

Figure 3 The Standard Distance of Restaurants in Built-up Area of Chuxiong City

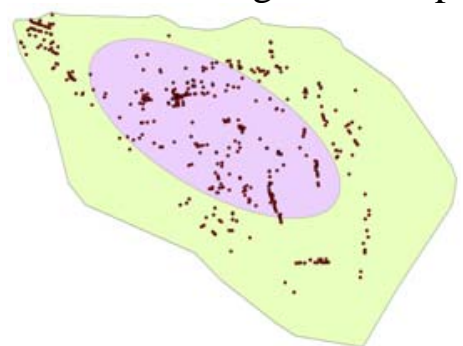

Figure 4 The Direction Distribution of Restaurants in Built-up Area of Chuxiong City

It could be seen from the figure 3 that a large number of restaurants were in the outside of the standard distance circle, space distribution of restaurants was discrete, they did not significantly concentrate in one place or a small area.

It could be observed in the figure 4 that direction distribution of restaurants was northwest southeast. The first reason was the trend of built-up area of Chuxiong city was northwest - southeast, this formed the overall pattern of it. The second reason was the ancient town of Yi nationality scenic spot rose in the northwest corner in recent years, many catering facilities aggregated there. 


\section{Study on the Distance of Restaurants and Roads, Lanes and Residential Areas in Built-up Area of Chuxiong City}

We studied the distance of restaurants and roads, lanes and residential areas in built-up area of Chuxiong city with buffer analysis, the key of buffer analysis was how to ascertain buffer size. Previous studies or used some values prescribed by the researcher, such as $50 \mathrm{~m}, 100 \mathrm{~m}$, $200 \mathrm{~m}[11,12,13]$ etc, or used minimum distance or maximum distance[14] to ineasure it. Obviously, those methods maybe not enough scientific. In order to promote the scientificity of ascertaining buffer size, this paper used clustering method.

In the first place, we computed the distance of every restaurant and road, lane and residential area in built-up area of Chuxiong city with ArcGIS near analysis tool respectively. Their statistical values were shown in table 1.

Table 1 The Statistical Values of the Distance

\begin{tabular}{|l|l|l|l|}
\hline & $\begin{array}{l}\text { the distance of } \\
\text { restaurants and roads }(\mathrm{m})\end{array}$ & $\begin{array}{l}\text { the distance of } \\
\text { restaurants and lanes } \\
(\mathrm{m})\end{array}$ & $\begin{array}{l}\text { the distance of } \\
\text { restaurants and } \\
\text { residential areas (m) }\end{array}$ \\
\hline minimum & 0.15 & 0.04 & 4.03 \\
\hline maximum & 287.33 & 324.38 & 698.82 \\
\hline total & 10918.30 & 72.53 & 84558.10 \\
\hline average & 22.19 & 63.91 & 171.87 \\
\hline standard deviation & 28.71 & 115.13 \\
\hline
\end{tabular}

According to table 1 it could be learned that, judging from the minimum distance, the distance of restaurants and lanes was nearest, the distance of restaurants and roads was middle, the distance of restaurants and residential areas was furthest. Judging from the maximum distance, the distance of restaurants and roads was nearest, the distance of restaurants and lanes was middle, the distance of restaurants and residential areas was furthest, the value was $698.82 \mathrm{~m}$. Judging from the average distance, the distance of restaurants and roads was nearest, the value was $22.19 \mathrm{~m}$, the distance of restaurants and lanes was middle, the value was $72.53 \mathrm{~m}$, the distance of restaurants and residential areas was furthest, the value was $171.87 \mathrm{~m}$, their proportion was 1: 3.27: 7.75. Obviously, restaurants centered around roads more. The main reason was the accessibility of restaurants that were near roads was better. These restaurants could attract more customers and gain more benefits.

Then, exporting the distance data from ArcMAP, and importing them to SPSS 22 software. So these could analyze by k-means clustering method with SPSS software.

K-means clustering is a method of vector quantization, originally from signal processing, that is popular for cluster analysis in data mining. K-means clustering aims to partition $\mathrm{n}$ observations into $\mathrm{k}$ clusters in which each observation belongs to the cluster with the nearest mean, serving as a prototype of the cluster. This results in a partitioning of the data space into Voronoi cells.

Given an initial set of $\mathrm{k}$ means $\mathrm{m} 1(1), \cdots, \mathrm{mk}(1)$ (see below), the algorithm proceeds by alternating between two steps[15]:

Assignment step: Assign each observation to the cluster whose mean yields the least within-cluster sum of squares (WCSS). Since the sum of squares is the squared Euclidean distance, this is intuitively the "nearest" mean. (Mathematically, this means partitioning the observations according to the Voronoi diagram generated by the means).

$$
S_{i}^{(t)}=\left\{x_{p}:\left\|x_{p}-m_{i}^{(t)}\right\|^{2} \leq\left\|x_{p}-m_{j}^{(t)}\right\|^{2} \forall j, 1 \leq j \leq k\right\}
$$

where each $x_{p}$ is assigned to exactly one $S^{(t)}$, even if it could be assigned to two or more of them. Update step: Calculate the new means to be the centroids of the observations in the new clusters.

$$
m_{i}^{(t+1)}=\frac{1}{\left|S_{i}^{(t)}\right|} \sum_{x_{j} \in S_{i}^{(t)}} x_{j}
$$

Since the arithmetic mean is a least-squares estimator, this also minimizes the within-cluster sum of squares (WCSS) objective. 
K-means clustering method needs specify the clustering number. Considering the maximum distance of restaurants and roads, lanes and residential areas(only 698.82m) and people's daily activities range, it was obviously not appropriate if too much clustering number was specified. So it was more logical if the clustering number was specified as three. The clustering results could be obtained with the above equations(table 2, table 3, table 4).

Table 2 The Clustering Result of the Roads

\begin{tabular}{|c|c|c|c|}
\hline & \multicolumn{3}{|c|}{ clustering } \\
\hline & 1 & 2 & 3 \\
\hline near_distance & 106.39 & 16.23 & 242.41 \\
\hline the number of cases of clustering & 25 & 464 & 3 \\
\hline ratio & $5.08 \%$ & $94.31 \%$ & $0.61 \%$ \\
\hline
\end{tabular}

Table 3 The Clustering Result of the Lanes

\begin{tabular}{|l|l|l|l|}
\hline \multicolumn{4}{|l|}{ clustering } \\
\hline & 1 & 2 & 3 \\
\hline near_distance & 36.47 & 218.87 & 120.84 \\
\hline the number of cases of clustering & 341 & 51 & 100 \\
\hline ratio & $69.31 \%$ & $10.37 \%$ & $20.33 \%$ \\
\hline
\end{tabular}

Table 4 The Clustering Result of the Residential Areas

\begin{tabular}{|l|l|l|l|}
\hline & \multicolumn{4}{l}{ clustering } & 3 \\
\hline & 1 & 2 & 90.72 \\
\hline near_distance & 400.91 & 212.37 & 263 \\
\hline the number of cases of clustering & 64 & 165 & $53.46 \%$ \\
\hline ratio & $13.01 \%$ & $33.54 \%$ & 3 and \\
\hline
\end{tabular}

Doing multiple ring buffer analysis with the buffering sizes from table 2, table 3 and table 4 respectively, then figure 5 .
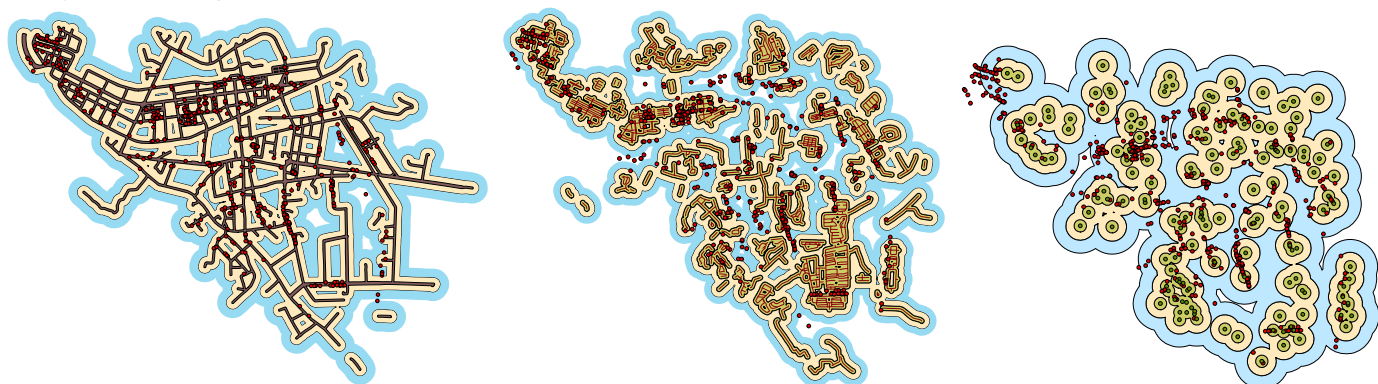

Figure 5 The Multiple Ring Buffer Diagram of the Roads, Lanes, Residential Areas

From the table 2, table 3, table 4 and figure 5, it could be seen that the most important clustering center of the distance of restaurants and roads was $16.23 \mathrm{~m}, 94.31 \%$ of the restaurants gathered around it. The most important clustering center of the distance of restaurants and lanes was $36.47 \mathrm{~m}, 69.31 \%$ of the restaurants gathered around it. The most important clustering center of the distance of restaurants and residential areas was $90.72 \mathrm{~m}, 53.46 \%$ of the restaurants gathered around it. The crowding level percentages of the restaurants were from top to low in a decreasing order. It could be shown that most of the restaurants centered around roads, while, crowding level to lanes and residential areas was relatively low.

Comparing the three clustering centers and the average distance of restaurants and roads, lanes, residential areas, it could be found that the deviation value of the most important clustering center of the distance of restaurants and roads and their average distance was $27 \%$, the deviation value of the most important clustering center of the distance of restaurants and lanes and their average distance 
was 50\%, the deviation value of the most important clustering center of the distance of restaurants and residential areas and their average distance was $47 \%$.

\section{Conclusions}

(1) The positions of the average center, median center and center element were located in the west of lucheng north road, north of lucheng west road, east of longhui road, and south of Zixi avenue in the old city zone. The center element was Kunming jianxin garden crossing-over bridge rice noodles shop (chuxiong shop).

(2) Space distribution of restaurants in Built-up area of Chuxiong city was discrete, they did not significantly concentrated in one place or a small area.

(3) Direction distribution of restaurants was northwest - southeast in Built-up Area of Chuxiong City.

(4) It could be found that most of the restaurants centered around roads, while, crowding level to lanes and residential areas was relatively low with k-means clustering method and buffer analysis.

\section{Acknowledgements}

This research was financially supported by Yunnan Excellent Young Teacher Project (natural geography) and Chuxiong Normal University key construction specialty Project(Human geography and urban and rural planning).

\section{References}

[1] Gang CHEN, zhenfang HUANG. Inquiry Into Economics Issues. Vol. 10 (2010), p. 129-134 (In Chinese).

[2] Weihong FENG, JinYun LI. Productivity Research. Vol. 2 (2012), p. 128-130 (In Chinese).

[3] Fei LI, Yuhua ZHANG. Commercial Economics Review. Vol. 5 (2005), p. 1-4+47 (In Chinese).

[4] Wenjing LI, Hongyi SONG, Dongqi LI. East China Economic Management. Vol. 2 (2011), p. 9-12 (In Chinese).

[5] Qingmei ZHAO. Economic Research Guide. Vol. 16 (2013), p. 131-135 (In Chinese).

[6] Zhiyi HU, Zhaogan ZHANG. Economics Geography. Vol. 1 (2002), p. 106-110 (In Chinese).

[7] Gang CHEN, Jianchun ZHANG. Journal of Hangzhou Normal University(Natural Science Edition). Vol. 2 (2011), p. 186-192 (In Chinese).

[8] Gang CHEN, Zhenfang HUANG. Tourism Tribune. Vol. 4 (2009), p. $61-66$ (In Chinese).

[9] Long DING, Huan ZHENG. Science Technology and Industry. Vol. 10 (2011), p. 73-75+112 (In Chinese).

[10]Guohua JIANG, Jun WU. ECONOMIC RESEARCH GUIDE. Vol. 2 (2009), p. 151-153 (In Chinese).

[11] Chen DAI. Modern Urban Research. Vol. 10 (2013), p.22-28 (In Chinese).

[12] Fangying ZHAO, Cuiping WANG, Ranran Song. Science \& Technology Information. Vol. 3(2012), p.51-53+57 (In Chinese).

[13] Zhongliang GAO, Ruliang ZHOU, Zhi LI, etc. Journal of Safety and Environment. Vol. 4(2012), p.243-246 (In Chinese).

[14] Hong MIAO, Min ZHANG. Journal of Arid Land Resources and Environment. Vol. 4(2014), p.179-186 (In Chinese). 
[15] MacKay, David. Information Theory, Inference and Learning Algorithms. Chapter, 20. Cambridge University Press(2003). 